\title{
Statistical study of M 51-type galaxies
}

\author{
S. A. Klimanov ${ }^{1}$ and V. P. Reshetnikov ${ }^{1,2}$ \\ 1 Astronomical Institute of St. Petersburg State University, 198504 St. Petersburg, Russia \\ 2 Isaac Newton Institute of Chile, St. Petersburg Branch, Russia \\ Received 23 August 2000 / Accepted 12 July 2001
}

\begin{abstract}
We present a statistical analysis of a new sample of M 51-type galaxies. Using the MCG and VV catalogues, we selected 32 such binary systems. We found that a typical M 51-type pair consists of a bright $L^{*}$ spiral galaxy and a satellite with blue luminosity $1 / 30-1 / 3$ of the primary one. The main galaxies in such pairs are often barred and have two well-defined spiral arms. M 51-type systems show an enhanced star formation rate (from FIR luminosities). We found a weak dependence of the star formation rate of the system on relative luminosity of the companion. M 51-type galaxies are relatively frequent: about 1/12 of all pairs are of M 51-type.
\end{abstract}

Key words. galaxies: evolution, general, interactions, photometry, peculiar, spiral

\section{Introduction}

Vorontsov-Velyaminov (1957) was one of the first to draw attention to an interesting subset of binary galaxies that consists of a large spiral galaxy and a relatively small companion near the end of the spiral arm of the primary one. Vorontsov-Veliaminov (1975) claimed that he selected 160 such M 51-type double systems. Many of them he included in his Atlas of Interacting Galaxies (1977). These objects turned out to be so numerous that Vorontsov-Veliaminov (1957) supposed that these galaxies are not the result of accidental projections but form physically connected systems. Further, he described the systems with two companions that are at the tips of two spiral arms. He suggested that small companions were formed in the main galaxies and separated and left afterwards.

Arp also studied this peculiar kind of double galaxy. He included 54 such systems in his Atlas of Peculiar Galaxies (1966). Arp speculated that small companions could have been thrown out from the nuclear regions of the parent galaxies.

Toomre \& Toomre (1972) believed that the appearance of M 51 is only as accidental projection of the smaller companion to the spiral arm of the main galaxy and in reality the satellite is $11 \mathrm{kpc}$ away from it. By means of numerical simulations with test particles they found that the satellite is moving on the streching orbit, almost perpendicularly to the plane of the main galaxy. Goward et al. (1990) obtained different parameters of the the orbit of the satellite. Unlike Toomre \& Toomre, they used self-gravitating particles and obtained the eccentricity of the orbit as $e \approx 0.1$ and inclination as $i \approx 50^{\circ}$. There were several works on

Send offprint requests to: V. P. Reshetnikov,

e-mail: resh@astro.spbu.ru numerical modelling of other M 51-type systems (for instance, NGC 7753/7752 - Salo \& Laurikainen 1993).

At present, a large amount of new data has been accumulated about galaxies that can belong to the M 51-type systems. On the whole it concerns apparent magnitudes, radial velocities, morphological types, and infrared data. As for satellites, because of their faintness (even $19^{\mathrm{m}}$ ), the specific data are very poor. Apparent magnitudes, radial velocities and morphological types are known only for a small number number of galaxies, apart from spectra and rotation curves.

Therefore, M 51-type galaxies are almost unexplored objects from the observational and theoretical points of view. We do not have even a good sample of such galaxies. M 51-type objects represent some of the best possible laboratories for studying the influence of small companions on the structure of spiral galaxies, on the possible generation of tidal spiral arms etc. The main aims of our work are to create a well-defined sample of M 51-type galaxies and to collect all available data about them in order to address the question of their origin.

\section{The sample of M 51-type galaxies}

Since Vorontsov-Velyaminov was the first to pick out this specific type of objects in a statistically sufficiently large group, we decided to consider his empirical definition as a model for M 51 galaxies. We can understand his real criteria through detailed examination of the candidates.

The main source of the sample was the Morphological Catalog of Galaxies (MCG) (1962-1968) by VorontsovVelyaminov et al. The author (VV) marked suspected M 51-type galaxies in the catalog. The catalog contains $\sim 30000$ galaxies and is essentially complete down to 
B-band magnitude 15.0 (e.g. Sharp 1986). Other than those, some systems were taken from the paper by Vorontsov-Velyaminov (1975) and from his Atlas of Interacting Galaxies (1977). In this way we selected 146 systems which were ranked by Vorontsov-Velyaminov according to M 51 type. Optical images of all double systems were extracted from the Digital Sky Survey ${ }^{1}$ (DSS), which is an electronic version of POSS. After that, we identified these images and collected available data about the objects from the NED and the LEDA databases.

Looking at the sample, we found that it is rather inhomogenious. In half the cases (74 objects) we found that with a high probability the suspected companion is the HII region or is a projected star. Also, we rejected systems with strongly disturbed structure or visible interaction with neighbouring galaxies.

To take into consideration possible influence of the environment, we calculated the dimensionless interaction parameter $Q=\left(d_{\mathrm{c}} d_{\mathrm{p}}\right)^{3 / 2} A^{-3}$ (Dahari 1984). Here $d_{\mathrm{c}}$ and $d_{\mathrm{p}}$ are the angular diameters of the M 51-type system main galaxy and of the nearest galaxy, and $A$ is their angular separation. This interaction parameter measures tidal effects and we have used it to characterize a degree of isolation of M 51-type galaxies.

As a result of our considerations we arranged a sample consisting of 32 pairs, which correspond to the criteria above in the best way - the sample G (consisting of the galaxies which we call "good"). This sample does not contain systems with interaction strength $Q_{1}=\log Q$ more than -0.8 . In order to test the statistical completeness of the sample, we applied the so-called $\left\langle V / V_{\max }\right\rangle$-test, which was used first for studies of the distribution of quasars by Schmidt (1968). For a uniform distribution in space, the mean value $\left\langle V / V_{\max }\right\rangle$ should be equal to $0.5 \pm 1 / \sqrt{12 \times n}$, where $n$ is the number of objects. For the sample $\mathrm{G}$ we find $\left\langle V / V_{\max }\right\rangle=0.46 \pm 0.05$ so that it appears complete. Considering the 19 systems with the $B$-band magnitude of the main galaxy $\leq 15.0$, we obtain $\left\langle V / V_{\max }\right\rangle=0.51 \pm 0.07$.

This sample of M 51-type objects partially overlaps with the Laurikainen et al. (1998) sample (13 systems), but for 4 objects only (Arp 82, Arp 83, Arp 86, Arp 87). The rest of the binary systems in the Laurikainen et al. sample ( 9 pairs) are not marked by Vorontsov-Velyaminov as M 51-type galaxies. Note also that the Laurikainen et al. sample is not complete in any sense.

We also distinguished an interesting case of systems that have two satellites at the tips of two spiral arms. For further investigations we arranged a sample consisting of 6 such galaxies - the sample TW (twice M 51-type). This sample does not contain systems with interaction parameter $Q_{1}=\log Q$ more than -1 . Due to the small volume of the TW sample we did not apply the $\left\langle V / V_{\max }\right\rangle$ test to it.

1 The Digitized Sky Surveys were produced at the Space Telescope Science Institute under U.S. Government grant NAG $\mathrm{W}-2166$.

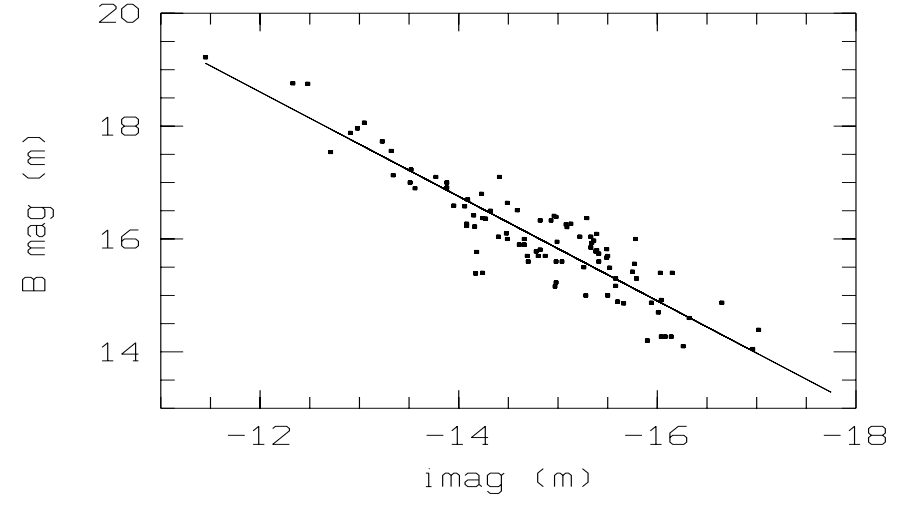

Fig. 1. The empirical relation between the "instrumental" magnitudes (imag) and total $B_{\mathrm{T}}$ magnitudes (mag). The points are data for Abell galaxies.

We collected available general observational characteristics of the samples in Appendix A and present their images in Appendix B (Figs. 7-9).

\section{Statistical analysis and discussion}

\subsection{General characteristics}

As a first step, we estimated the apparent $B$ magnitudes that were unknown for the majority of the satellites. We used an empirical relation between the "instrumental" apparent magnitude from the DSS image of a galaxy and its total $B$ magnitude for such estimates. To create the empirical relation, we considered several Abell clusters of galaxies with published photometry and compared the $B$-band magnitudes of galaxies and their "instrumental" magnitudes defined as $-2.5 \log D_{\Sigma}$, where $D_{\Sigma}$ is the sum of all pixel values within the galaxy image above the $2 \sigma_{\mathrm{b}}$ of the background $\left(\sigma_{\mathrm{b}}-\right.$ standard deviation of the background level). As a result, the galaxies grouped along a linear dependence (Fig. 1): $B_{\mathrm{T}}=0.925 \cdot \mathrm{imag}+29.71$. The standard deviation of the objects relative to the best fit line is 0.43 . The derived relation reproduces the main galaxy magnitudes (taken from the LEDA) with mean difference $\langle B(\mathrm{DSS})-B($ main $)\rangle=+0.14$ and $\sigma=0.42$. But if we take into account that almost all Abell galaxies have $B_{\mathrm{T}}>14^{\mathrm{m}}$ (see Fig. 1) and exlude main galaxies with brighter magnitudes, the mean difference between $B$ (DSS) and $B$ (main) will be 0.01 with scatter 0.30 . The results of our measurements for all companions are presented in the Tables 9-10.

To obtain absolute $B$ magnitudes we used radial velocities corrected for Local Group infall onto Virgo cluster, which are given in the LEDA database. Using these velocities and apparent $B$ magnitudes obtained either from the LEDA or from our empirical dependence, we calculated absolute $B$ magnitudes for the main galaxies and the satellities $\left(H_{0}=75 \mathrm{~km} \mathrm{~s}^{-1} / \mathrm{Mpc}\right)$. These magnitudes were corrected for the Galactic extinction according to NED. Due to disturbed morphology of many sample objects (see Figs. 7-9) and, therefore, uncertain inclinations, we did not apply correction for internal absorption. This corection is not significant for the most of 
Table 1. Absolute magnitudes of the M 51 and CPG pairs.

\begin{tabular}{|c|c|c|c|c|c|}
\hline Sample & $N$ & $\left\langle M_{B}\right\rangle_{\mathrm{m}}$ & $\sigma_{\mathrm{m}}$ & $\left\langle M_{B}\right\rangle_{\mathrm{s}}$ & $\sigma_{\mathrm{s}}$ \\
\hline CPG & 1170 & -20.57 & 1.13 & -19.91 & 1.32 \\
M 51 (G) & 28 & -20.02 & 1.26 & -17.77 & 1.94 \\
M 51 (TW) & 5 & -19.35 & 1.74 & -17.08 & 2.28 \\
\hline
\end{tabular}

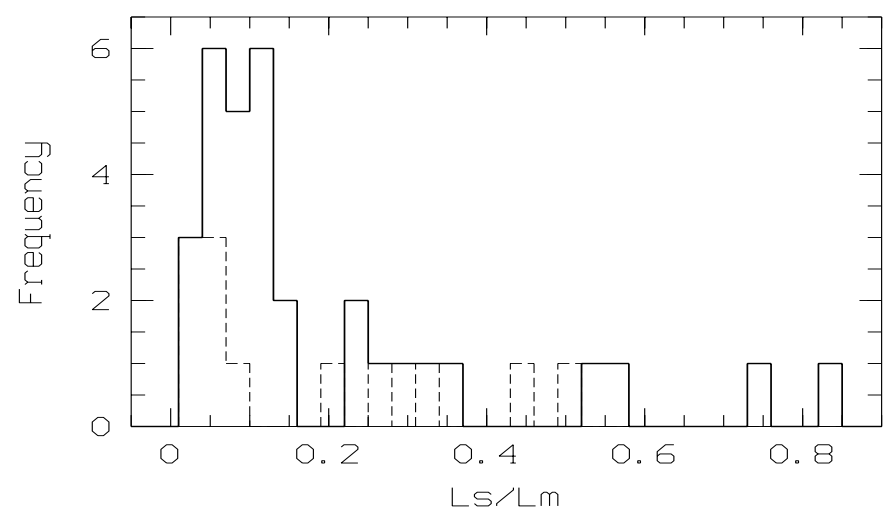

Fig. 2. The distribution of the $L_{\mathrm{s}} / L_{\mathrm{m}}$ ratio. Solid line presents the data for the G sample, dashed - for the TW sample.

our objects. According to our measurements and the RC3 (de Vaucouleurs et al. 1991) data, the mean axial ratio is $b / a=0.6$. Therefore, the mean value of the internal absorption is $A(B)=1.5 \cdot \log (a / b)=0 . \cdot 3$ (according to RC3 for an Sc galaxy). This value is comparable with the typical error of the absolute magnitude.

The mean absolute magnitudes $\left\langle M_{B}\right\rangle$ and corresponding standard deviations $\sigma$ for the main galaxies and the satellities are compared with the CPG pairs (Karachentsev 1972, 1987) in Table 1. As one can see, the main components of our M 51-type pairs have comparable absolute magnitudes with those for $\mathrm{CPG}$ galaxies. Moreover, the mean absolute magnitude $\left\langle M_{B}\right\rangle_{\mathrm{m}}=-20.0$ is close to the Milky Way magnitude and to the characteristic $L^{*}$ luminosity in the Schechter function. As for the satellites, they have, on average, significantly fainter magnitudes in comparison with CPG secondary components. This could be explained by an effect of selection since we selected the systems with the relatively faint satellites only.

The distribution of the companion to main galaxy luminosity ratio $\left(L_{\mathrm{s}} / L_{\mathrm{m}}\right)$ is shown in Fig. 2. The distribution for the G sample is quite wide with $\left\langle L_{\mathrm{s}} / L_{\mathrm{m}}\right\rangle=0.19 \pm$ $0.21(\sigma)$ The TW sample gives $\left\langle L_{\mathrm{s}} / L_{\mathrm{m}}\right\rangle=0.17 \pm 0.18(\sigma)$ The corresponding median values are 0.10 and 0.08 for the $\mathrm{G}$ and $\mathrm{TW}$ samples correspondingly.

Most of the satellites (28/32 or $88 \%$ ) have relative luminosities between 0.02 and 0.36 . This allows us to suggest a formal criterion to separate M 51-type systems: $M$ 51-type galaxies are double systems with a relatively faint companion (with luminosity between $1 / 30$ and $1 / 3$ of the main galaxy) near the end of spiral arm of the primary galaxy. What does "near the end" mean? According to our measurements (see item 3.4 and Fig. 3), the
Table 2. Morphological types of M 51 primary galaxies.

\begin{tabular}{|c|c|c|c|c|c|}
\hline Sample & $N$ & $a+a b(\%)$ & $b(\%)$ & $b c(\%)$ & $c(\%)$ \\
\hline M 51(G) & 32 & 3 & 16 & 28 & 53 \\
M 51 (TW) & 5 & - & 60 & - & 40 \\
\hline Sample & $N$ & $a+a b(\%)$ & $b+b c(\%)$ & $c+d+m(\%)$ \\
\hline VV & 149 & 25 & \multicolumn{2}{|c|}{36} & 39 \\
\hline
\end{tabular}

Table 3. Frequency of spiral arms in M 51 main galaxies.

\begin{tabular}{|c|c|c|c|c|c|c|}
\hline Sample & $N$ & $\begin{array}{c}1 \text { arm } \\
(\%)\end{array}$ & $\begin{array}{c}2 \\
(\%)\end{array}$ & $\begin{array}{c}3 \\
(\%)\end{array}$ & $\begin{array}{c}4 \\
(\%)\end{array}$ & $\begin{array}{c}5 \\
(\%)\end{array}$ \\
\hline M 51(G) & 27 & 4 & 74 & 14 & 4 & 4 \\
M 51 (TW) & 6 & - & 100 & - & - & - \\
\hline
\end{tabular}

Table 4. Bars frequences in M 51 samples.

\begin{tabular}{|c|c|c|c|}
\hline Sample & $N$ & Barred (\%) & Unbarred (\%) \\
\hline M 51 (G) & 32 & 56 & 44 \\
VV & 149 & 32 & 68 \\
\hline
\end{tabular}

projected companion separations should be less than 2 main galaxy diameters.

\subsection{Morphological types}

Morphological types of main galaxies from the NED and LEDA databases are summarized in Table 2. For the comparison, the data for the Vorontsov-Velyaminov's (1975) sample of 149 M 51-type systems are also presented (sample VV).

Early spirals (Sa or Sab) are almost absent in our samples. The reasons of that are not clear. It could be an effect of selection because we preferably selected galaxies with distinct spiral structure.

Table 3 presents the distribution of the primary galaxies according to the number of spiral arms. For the classification we used the DSS images of the systems. The grand-design spirals with two arms dominate (almost 3/4) among the M 51-type galaxies.

Note that nearly face-on galaxies dominate in our sample (see Figs. 7-9) so the influence of inclination on the morphological type and the number of arms estimations is neglible.

\subsection{Frequency of barred galaxies}

In order to investigate the bar frequency among M 51-type primary galaxies, we extracted morphological information from the LEDA and NED. The fractions of barred and non-barred galaxies (in percentages) are listed in Table 4, where we included also the data for 149 M 51-type galaxies from Vorontsov-Velyaminov (1975).

The percent of barred galaxies in the $\mathrm{G}$ sample is $56 \% \pm 13 \%$ (Poisson error). This fraction exeeds the fraction of field barred galaxies in the optical $(\sim 30 \%-$ de Vaucouleurs 1963; Eskridge et al. 2000) and is consistent with the fraction of barred galaxies among double 
Table 5. Relative distance of the companion.

\begin{tabular}{|c|c|c|c|c|c|}
\hline Sample & $N$ & $\langle S\rangle$ & $\sigma_{S}$ & $\langle 1 / S\rangle$ & $\sigma_{1 / S}$ \\
\hline $\mathrm{G}$ & 32 & 0.86 & 0.35 & 1.34 & 0.48 \\
$\mathrm{TW}$ & 6 & 0.86 & 0.21 & 1.22 & 0.29 \\
\hline
\end{tabular}

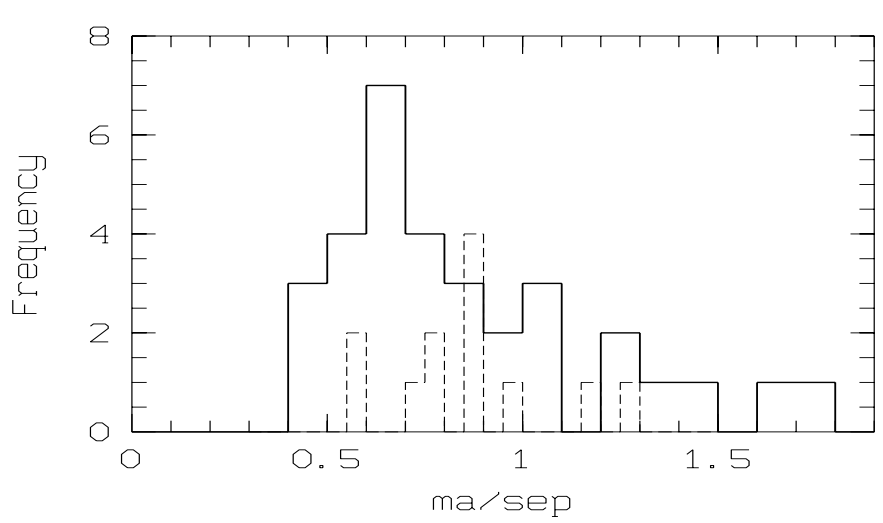

Fig. 3. The distribution of the semimajor axis to angular separation ratio (ma/sep). The $\mathrm{G}$ sample is shown by solid line, sample TW - dashed.

systems (Elmegreen et al. 1990). The VorontsovVelyaminov sample is contaminated by a large number of non-M 51-type objects and does show only $\sim 1 / 3$ barred galaxies.

\subsection{Satellites' distances}

The satellites are situated at different distances from the main galaxies. To obtain the distribution of these distances we derived the semimajor axis to angular separation ratio denoted by $S$ as well as inverse value $1 / S$ using the DSS images of systems. The mean values and standard deviations are listed in Table 5 (see also Fig. 3).

Thus the satellites in M 51-type systems are situated near the boundary of the stellar disks of the main galaxies. According to Lin \& Tremaine (1983) the simple estimate of the merging time of a satellite with main galaxy is $t_{\text {merge }} \approx(0.3-1) \times 10^{9}$ years, or less than 0.1 of the Hubble time. It might suppose that such close systems cannot exist for a long time (more than several orbital periods) and therefore the satellites of M 51-type systems cannot have a common origin with the main galaxies and were captured by the central galaxies relatively recently.

Pasha et al. (1988) supported the possible existence of almost circular stationary orbits of satellites in close double systems. According to this work the radius of the stable orbit falls in a narrow interval $1.3 \pm 0.1 R$, where $R$ is the radius of the stellar disk. As is seen in Table 5 the mean value of angular separation to semimajor axis ratio is close to this value. Because of the roughness of the measurements and large dispersions it cannot show the existence of the stationary stable orbits and more precise measurements should be undertaken using detailed images of the galaxies.

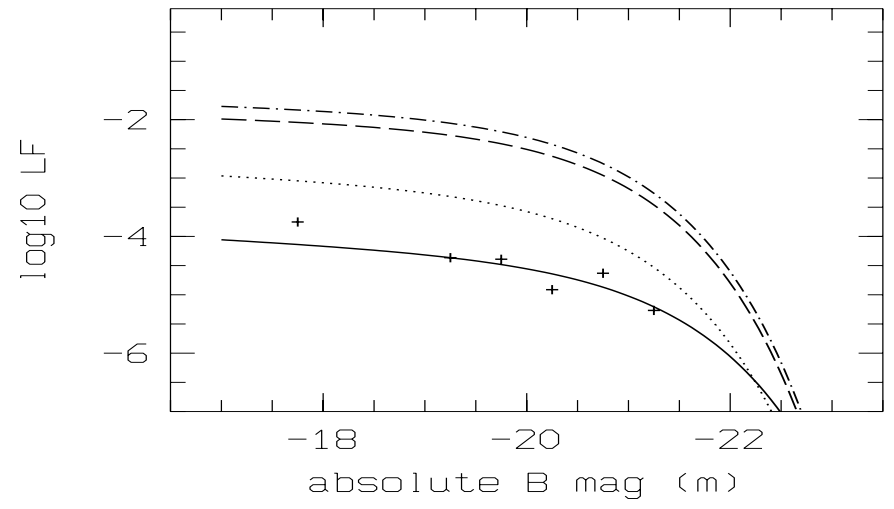

Fig. 4. The optical luminosity functions of the samples. Solid line corresponds to the sample $G_{\text {main }}$ (excluding point at $M_{B}=$ -17.75), dotted line - to the sample CPG, dashed line - to the sample "Spiral", dash-dot-dash line - to the sample "All". Crosses present the M 51-type main galaxies $\left(G_{\text {main }}\right)$ data.

\subsection{Luminosity function}

We undertook an attempt to derive the optical luminosity function (OLF) for the main components of M 51-type systems. We extracted the main components in a separated sample $\left(G_{\text {main }}\right)$ and assumed that these galaxies are uniformly distributed in space. To obtain the OLF we used the standard expression (e.g. Felten 1976):

$\phi(M)=\frac{4 \pi}{\Omega} \frac{\xi}{\Delta M} \sum_{i} \frac{1}{V_{\mathrm{m}}\left(M_{i}\right)}$.

Here $\phi(M)$ is the differential luminosity function giving the number of galaxies per unit $\mathrm{d} V$ and per unit $\mathrm{d} M . \Omega$ is the effective solid angle covered by the sample, $\xi$ is the parameter that corrects OLF for incompleteness. For $\xi$ calculation we used the procedure described by Huchra \& Sargent (1973). $V_{\mathrm{m}}\left(M_{i}\right)$ is the volume of a sphere with the radius that is the maximum distance a source of absolute magnitude $M_{i}$ could been detected in a survey down to $m_{\lim }$. The summation is over all galaxies in the absolute magnitude interval $M+0.5 \Delta M \geq M_{i}>M-0.5 \Delta M$. After investigation we chose $\Delta M=0.5$ and $m_{\text {lim }}=15.0$. The derived OLF was fitted by the Schechter function:

$\phi_{\mathrm{sch}}(M)=\phi_{*} 10^{0.4(\alpha+1)\left(M_{*}-M\right)} \exp \left(-10^{0.4\left(M_{*}-M\right)}\right)$,

where $\phi_{*}, M_{*}$ and $\alpha$-parameters.

We compared our results with three other samples. The first contains galaxies of all Hubble types (sample "All"), the second contains only spiral galaxies (sample "Spiral"). The data for both samples were taken from Marzke et al. (1998). The third one consists brighter components of CPG pairs (sample "CPG") and was taken from Xu \& Sulentic (1991). The data for all samples are presented in Table 6 , where the two last columns give $\rho$ - the space density (in $\mathrm{Mpc}^{-3}$ ) and $r_{\rho}$ - the space density of galaxies of the sample $G_{\text {main }}$ to space density of galaxies of other sample ratio. Fitted OLF for all described samples are plotted in Fig. 4.

We note that in spite of a few points in our sample the slope of our curve is similar to other ones. As is seen in 
Table 6. Parameters of optical luminosity functions.

\begin{tabular}{|c|c|c|c|c|c|c|c|c|c|}
\hline Sample & $N$ & $m_{\lim }$ & $\Omega$ & $\xi$ & $\phi_{*}$ & $\alpha$ & $M_{*}$ & $\rho$ & $r_{\rho}$ \\
\hline$G_{\text {main }}$ & 19 & 15.0 & 11.98 & 3.79 & $4.38 \times 10^{-5}$ & -1.22 & -20.60 & $2.9 \times 10^{-4}$ & - \\
CPG & 491 & 15.6 & 4.38 & 2.6 & $6.51 \times 10^{-4}$ & -1.2 & -20.1 & $3.4 \times 10^{-3}$ & 0.09 \\
Spiral & 3227 & - & - & - & $3.38 \times 10^{-3}$ & -1.11 & -20.05 & $1.5 \times 10^{-2}$ & 0.02 \\
All & 5036 & - & - & - & $5.40 \times 10^{-3}$ & 1.12 & -20.05 & $2.4 \times 10^{-2}$ & 0.01 \\
\hline
\end{tabular}

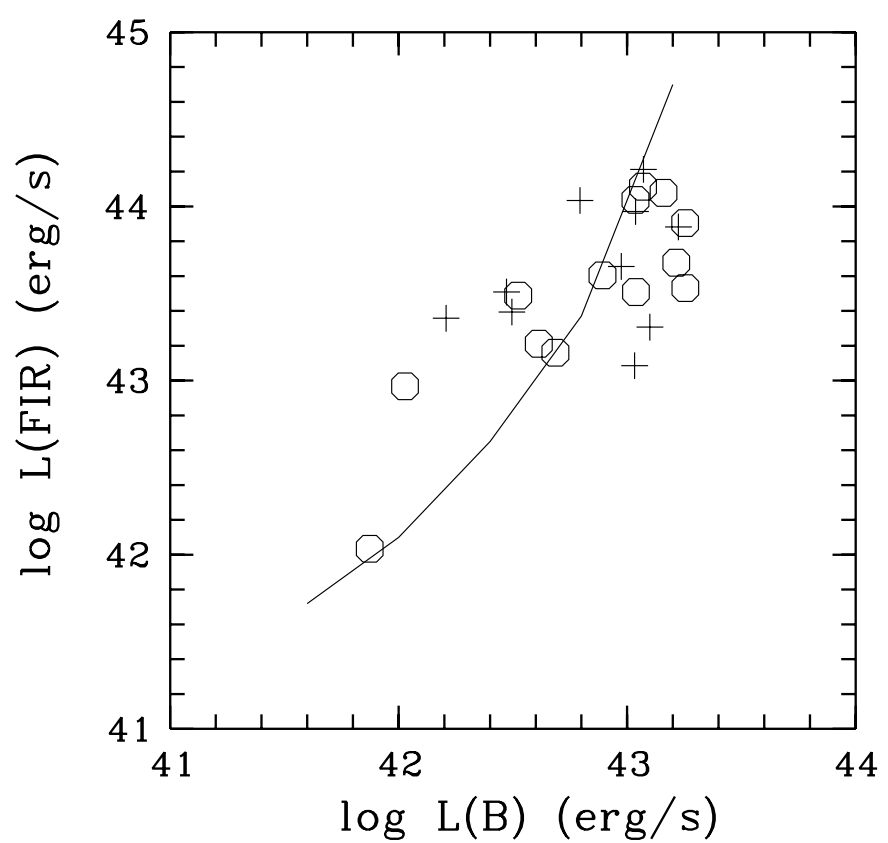

Fig. 5. Plot of the FIR luminosity of M 51-type main galaxies versus total blue luminosity. The solid line is the fit for interacting galaxies from Bushouse et al. (1988). Crosses are for the systems with $Q_{1}>-2.6$, circles - for the systems with $Q_{1}<-2.6$.

Table 6 , the space density of the galaxies in the sample $G_{\text {main }}$ is nearly twelve times lower than the space density of the CPG brighter components. Hence, we conclude that one twelfth part of the CPG pairs consists of systems with faint components that have nearly ten times less luminosity than brighter components (or about $1 \%$ of all galaxies). This rough estimate shows that M 51-type galaxies are not extremly rare objects among double systems.

\subsection{Star formation}

We extracted the IRAS survey data from the NED and estimated far-IR (FIR) luminosities for most of our objects (Tables 9-10). It is assumed that IR-emission of a galaxy arises from heating of the interstellar dust associated with star-forming regions. FIR flux can be obtained using the standard formula (e.g. Keel 1993):

$$
F_{\mathrm{FIR}}=1.26 \times 10^{-14}\left(2.58 S_{60}+S_{100}\right) \quad\left(\mathrm{W} / \mathrm{m}^{2}\right),
$$

where $S_{60}$ and $S_{100}$ - are the fluxes (in Jy) at 60 and $100 \mu \mathrm{m}$. Since the fluxes were known for $75 \%$ of galaxies in the sample $\mathrm{G}$ and for two galaxies only in the sample TW, we have made our calculations for the first sample only.

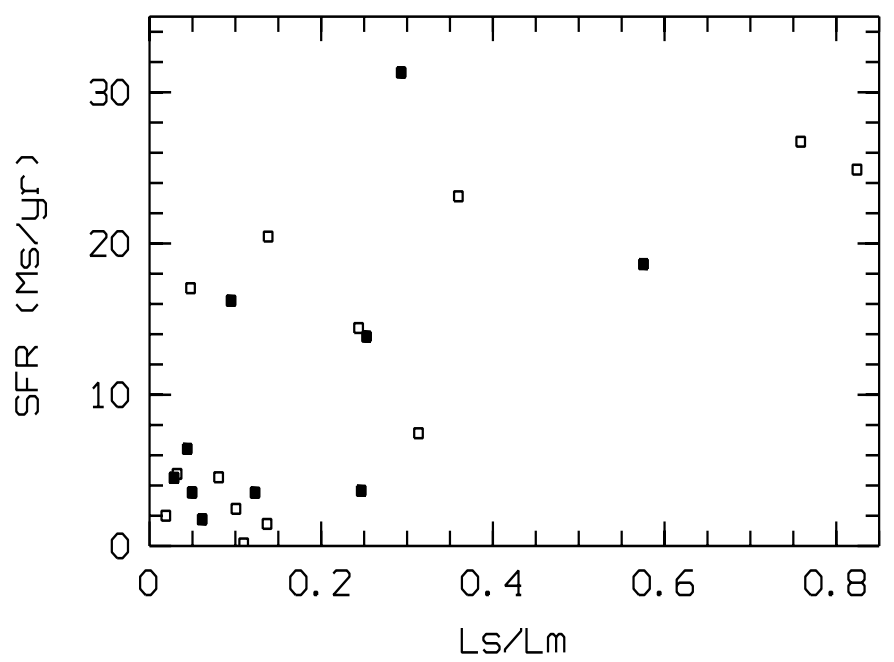

Fig. 6. The dependence of $S F R$ on the luminosity of satellite to luminosity of main galaxy ratio $(L \mathrm{~s} / L \mathrm{~m})$. Filled squares are for systems with $Q_{1}>-2.6$, unfilled ones - for systems with $Q_{1}<-2.6$.

We estimated star formation rate (SFR) as well as $L_{\mathrm{FIR}} / L_{B}$ ratio using formulae from Bushouse (1987) and Keel (1993):

$S F R=1.34 \times 10^{-43} L_{\mathrm{FIR}} \quad\left(M_{\odot} / \mathrm{yr}\right)$,

$L_{\mathrm{FIR}} / L_{B}=4.16 \times 10^{-7} \times 10^{0.4 m_{B}}\left(2.58 S_{60}+S_{100}\right)$,

where $m_{B}$ - apparent $B$ magnitude of galaxy.

Since the IRAS survey has low angular resolution, $S F R$ and $L_{\mathrm{FIR}} / L_{B}$ values correspond to the whole system without separation of individual pair members. Derived mean and median (in round brackets) values and standard deviations are:

$\left\langle L_{\mathrm{FIR}}\right\rangle=2.1 \times 10^{10} L_{\odot}\left(1.2 \times 10^{10}\right), \sigma_{L_{\mathrm{FIR}}}=1.8 \times 10^{10} L_{\odot}$ (23 galaxies);

$\langle S F R\rangle=11.0 M_{\odot} / \mathrm{yr}(6.4), \sigma_{S F R}=9.5 M_{\odot} / \mathrm{yr}(23$ galaxies);

$\left\langle L_{\mathrm{FIR}} / L_{B}\right\rangle=1.3(1.23), \sigma_{L}=0.8$ (24 galaxies).

After correction of the total FIR-luminosity for the contribution of the satellite (using the $L_{\mathrm{s}} / L_{\mathrm{m}}$ ratio in the $B$ passband), we obtain the next values for the main galaxies only: $\langle S F R\rangle=9 M_{\odot} / \mathrm{yr},\left\langle L_{\mathrm{FIR}} / L_{B}\right\rangle=1.1$.

Bushouse (1987) studied his sample of interacting galaxies and obtained close mean value of $S F R-$ $7.2 M_{\odot} /$ yr. At the same time, $S F R$ for isolated galaxies founded by him are approximately 6 time less. Thus, the mean value of $S F R$ for M 51-type galaxies is significant. The mean value of $L_{\mathrm{FIR}} / L_{B}$ for our galaxies (1.1) is also much higher than for isolated spirals. For instance, the mean value of $L_{\mathrm{FIR}} / L_{B}$ for Sa-bc and Sc-d spirals is 
Table 7. Catalog of M 51-type galaxies - the initial data.

\begin{tabular}{|c|c|c|c|c|c|c|c|c|}
\hline \multicolumn{9}{|c|}{ GALAXIES OF TYPE M 51 (THE SAMPLE G) } \\
\hline$N$ & MCG & Names & RA ( 1950.0$)$ & $\operatorname{DEC}(1950.0)$ & $V_{\mathrm{r}}$ & $A_{B}$ & $B_{\mathrm{T}}$ & Type \\
\hline 1 & $-1-1-70$ & & 001501.70 & -045420.0 & $5985 \pm 42$ о & 0.14 & 14.89 & $\mathrm{Sc}(1)$ \\
\hline 2 & $-2-2-54$ & $\begin{array}{l}\text { ngc } 0151 \\
\text { ngc } 0153\end{array}$ & 003130.10 & -095856.0 & $\begin{array}{l}3712 \pm 49 \text { o } \\
3747 \pm 7 \mathrm{~m}\end{array}$ & 0.14 & 12.25 & $\mathrm{SB}(\mathrm{r}) \mathrm{bc}$ \\
\hline 3 & & $\begin{array}{c}\text { ngc } 0646 \text { a } \\
\text { vv443 a } \\
\text { eso080-ig002 a } \\
\text { ngc } 0646 \text { b } \\
\text { vv } 443 \text { b } \\
\text { eso080-ig002 b }\end{array}$ & $\begin{array}{l}013546.00 \\
013555.00\end{array}$ & $\begin{array}{l}-650900.0 \\
-650904.0\end{array}$ & $8112 \pm 91$ o & $\begin{array}{l}0.12 \\
0.12\end{array}$ & 14.32 & $\mathrm{SBc}(\mathrm{l})$ \\
\hline 4 & $\begin{array}{l}6-5-78 \mathrm{a} \\
6-5-78 \mathrm{~b}\end{array}$ & $\begin{array}{l}\text { ngc } 0797 \mathrm{a} \\
\text { ugc } 01541 \mathrm{a} \\
\text { vv } 428 \mathrm{a} \\
\text { ngc } 0797 \mathrm{~b} \\
\text { ugc } 01541 \mathrm{~b} \\
\text { vv } 428 \mathrm{~b}\end{array}$ & $\begin{array}{l}020027.706 \\
020024.36\end{array}$ & $\begin{array}{l}375237.50 \\
\\
375220.2\end{array}$ & $\begin{array}{l}5600 \pm 52 \text { o } \\
5631 \pm 33 \mathrm{~m}\end{array}$ & $\begin{array}{l}0.24 \\
0.24\end{array}$ & $\begin{array}{l}13.87 \\
16.9\end{array}$ & $\begin{array}{c}\mathrm{SAB}(\mathrm{s}) \mathrm{a} \\
\mathrm{E}\end{array}$ \\
\hline 5 & $-1-7-7$ & $\begin{array}{l}\operatorname{arp} 054 \\
\text { vv } 453\end{array}$ & 022131.4 & -045512.0 & $12992 \pm 60$ o & 0.12 & 15.02 & $\mathrm{SBc}(\mathrm{l})$ \\
\hline 6 & $\begin{array}{l}-4-9-17 \mathrm{a} \\
-4-9-17 \mathrm{~b}\end{array}$ & $\begin{array}{c}\text { ngc } 1347 \text { a } \\
\text { eso548-g027 a } \\
\text { arp } 039 \text { a } \\
\text { vv } 023 a \\
\text { ngc } 1347 \text { b } \\
\text { eso548-g027 b } \\
\text { arp } 039 \text { b } \\
\text { vv } 023 b\end{array}$ & $\begin{array}{l}032730.00 \\
\\
032730.00\end{array}$ & $\begin{array}{l}-222700.0 \\
-222700.0\end{array}$ & $\begin{array}{l}1775 \pm 30 \mathrm{o} \\
1760 \pm 9 \mathrm{~m}\end{array}$ & $\begin{array}{l}0.15 \\
\\
\\
0.15\end{array}$ & 13.90 & $\mathrm{SB}(\mathrm{s}) \mathrm{c}:$ pec \\
\hline 7 & $\begin{array}{l}4-20-4 \\
4-20-5\end{array}$ & $\begin{array}{c}\text { ngc } 2535 \\
\text { ugc } 04264 \text { a } \\
\text { arp } 082 \text { a } \\
\text { vv } 009 \mathrm{a} \\
\text { ngc } 2536 \\
\text { ugc } 04264 \\
\text { arp } 082 \text { b } \\
\text { vv } 009 b\end{array}$ & 080815.80 & $\begin{array}{l}252123.0 \\
251943.0\end{array}$ & $\begin{array}{l}4084 \pm 30 \mathrm{o} \\
4097 \pm 7 \mathrm{~m} \\
4142 \pm 62 \mathrm{o} \\
4103 \pm 9 \mathrm{~m}\end{array}$ & 0.18 & 14.87 & $\begin{array}{l}\mathrm{SA}(\mathrm{r}) \mathrm{c} \text { pec } \\
\mathrm{SB}(\mathrm{rs}) \mathrm{c} \text { pec }\end{array}$ \\
\hline 8 & $-2-22-26$ & vv 421 & 083949.93 & -134045.8 & & 0.23 & 15.21 & S (l) \\
\hline 9 & $1-24-20$ & ngc 2864 & 092137.20 & 060922.0 & $3546 \pm 15$ о & 0.21 & 15.27 & Sc (l) \\
\hline 10 & $10-14-20$ & $\begin{array}{l}\text { ugc } 05077 \\
\text { vv } 464\end{array}$ & 092907.303 & 595758.82 & $\begin{array}{c}12128 \pm 110 \mathrm{o} \\
11987 \pm 9 \mathrm{~m}\end{array}$ & 0.12 & 15.28 & $\mathrm{SBb}$ \\
\hline 11 & $-3-28-1$ & vv 410 & 104139.80 & -161228.0 & $9550 \pm 43$ о & 0.21 & 14.35 & $\mathrm{SAB}(\mathrm{rs}) \mathrm{c}$ pec? \\
\hline
\end{tabular}


Table 7. continued.

\begin{tabular}{|c|c|c|c|c|c|c|c|c|}
\hline \multicolumn{9}{|c|}{ GALAXIES OF TYPE M 51 (THE SAMPLE G) } \\
\hline 12 & $\begin{array}{l}7-23-39 a \\
7-23-39 \mathrm{~b}\end{array}$ & $\begin{array}{l}\text { ugc } 06293 \text { a } \\
\text { ugc } 06293 \text { b }\end{array}$ & $\begin{array}{lll}11 & 13 & 43.97 \\
11 & 13 & 40.15\end{array}$ & $\begin{array}{lll}41 & 21 & 04.8 \\
41 & 20 & 49.5\end{array}$ & $6431 \pm 60$ о & $\begin{array}{l}0.06 \\
0.06\end{array}$ & 16.44 & Scd \\
\hline 13 & $\begin{array}{l}3-30-39 \\
3-30-37\end{array}$ & $\begin{array}{l}\text { ngc } 3800 \\
\text { ugc } 06634 \\
\text { arp } 083 \text { a } \\
\text { vv } 350 \mathrm{a} \\
\text { ngc } 3799 \\
\text { ugc } 06630 \\
\text { arp } 083 \text { b } \\
\text { vv } 350 \mathrm{~b}\end{array}$ & $\begin{array}{r}113737.80 \\
\\
113734.00\end{array}$ & $\begin{array}{l}153712.0 \\
\\
153616.0\end{array}$ & $\begin{array}{c}3474 \pm 94 \mathrm{o} \\
3306 \pm 15 \mathrm{~m} \\
3462 \pm 114 \mathrm{o} \\
3312 \pm 6 \mathrm{~m}\end{array}$ & 0.13 & 13.75 & $\begin{array}{c}\text { SAB(rs)b: } \\
\text { pec } \\
\text { SB(s)b: } \\
\text { pec }\end{array}$ \\
\hline 14 & $\begin{array}{l}4-28-21 \\
4-28-20\end{array}$ & $\begin{array}{l}\text { ngc } 3808 \mathrm{~A} \\
\text { ugc } 06643 \text { a } \\
\text { arp } 087 \mathrm{a} \\
\text { vv } 300 \mathrm{a} \\
\text { ngc } 3808 \mathrm{~B} \\
\text { ugc } 06643 \mathrm{~b} \\
\operatorname{arp} 087 \mathrm{~b} \\
\text { vv } 300 \mathrm{~b}\end{array}$ & $\begin{array}{r}113807.75 \\
\\
113808.12\end{array}$ & $\begin{array}{llll}22 & 42 & 27.3 \\
& & & \\
22 & 4328.6\end{array}$ & $\begin{array}{l}7050 \pm 37 \text { o } \\
7076 \pm 6 \mathrm{~m} \\
7189 \pm 55 \mathrm{o}\end{array}$ & 0.11 & 14.40 & $\begin{array}{c}\text { SAB(rs)c: } \\
\text { pec } \\
\text { I0? pec }\end{array}$ \\
\hline 15 & $\begin{array}{l}7-25-2 \mathrm{a} \\
7-25-2 \mathrm{~b}\end{array}$ & $\begin{array}{l}\text { ugc } 06865 \\
\text { arp } 062 \text { a } \\
\text { vv } 286 \mathrm{a} \\
\text { ugc } 06865 \\
\text { arp } 062 \text { b } \\
\text { vv } 286 \mathrm{~b}\end{array}$ & $\begin{array}{r}115100.00 \\
115100.00\end{array}$ & $\begin{array}{l}4344.00 .0 \\
434400.0\end{array}$ & $\begin{array}{l}5885 \pm 102 \mathrm{o} \\
5886 \pm 09 \mathrm{~m}\end{array}$ & $\begin{array}{l}0.09 \\
0.09\end{array}$ & 14.42 & $\begin{array}{c}\mathrm{SB} \\
\mathrm{E}(\mathrm{c})\end{array}$ \\
\hline 16 & $9-20-89$ & $\begin{array}{c}\text { ngc } 4088 \\
\text { ugc } 07081 \\
\text { arp } 018 \\
\text { vv } 357\end{array}$ & 120302.00 & 504903.0 & $\begin{array}{l}728 \pm 51 \text { o } \\
756 \pm 7 \mathrm{~m}\end{array}$ & 0.09 & 11.16 & $\mathrm{SAB}(\mathrm{rs}) \mathrm{bc}$ \\
\hline 17 & $7-25-33$ & $\begin{array}{c}\text { ngc } 4137 \\
\text { ugc } 07135 \\
\text { vv } 454\end{array}$ & 120646.08 & 442204.0 & $10366 \pm 954$ o & 0.06 & 15.36 & $\mathrm{SB}(\mathrm{rs}) \mathrm{c} ?$ \\
\hline 18 & $-2-31-23$ & ngc 4188 & 121132.80 & -121831.0 & $4257 \pm 36$ о & 0.21 & 14.54 & Sc $(1)$ \\
\hline 19 & $1-31-40$ & $\begin{array}{c}\text { ic } 3115 \\
\text { ugc } 07333 \\
\text { vv } 431\end{array}$ & 121526.72 & 065552.9 & $\begin{array}{l}733 \pm 60 \mathrm{o} \\
733 \pm 6 \mathrm{~m}\end{array}$ & 0.10 & 14.07 & $\mathrm{SB}(\mathrm{s}) \mathrm{cd}$ \\
\hline 20 & $7-27-12$ & $\begin{array}{c}\text { ic } 4056 \\
\text { ugc } 08126 \\
\text { vv } 418\end{array}$ & 125824.00 & 400100.0 & & 0.07 & 16.00 & $\mathrm{SBbc}$ \\
\hline
\end{tabular}


Table 7. continued.

\begin{tabular}{|c|c|c|c|c|c|c|c|c|}
\hline \multicolumn{9}{|c|}{ GALAXIES OF TYPE M 51 (THE SAMPLE G) } \\
\hline 21 & $\begin{array}{l}8-25-12 \\
8-25-14\end{array}$ & $\begin{array}{c}\text { m 051a } \\
\text { ngc } 5194 \\
\text { ugc } 08493 \\
\text { arp 085 a } \\
\text { vv 001a } \\
\text { vv } 403 \\
\text { m } 051 \mathrm{~b} \\
\text { ngc } 5195 \\
\text { ugc } 08494 \\
\text { arp } 085 \text { b } \\
\text { vv } 001 b\end{array}$ & 132745.98 & 473132.0 & $\begin{array}{c}462+/-29 \text { o } \\
461 \pm 12 \mathrm{~m} \\
\\
565 \pm 40 \mathrm{o} \\
471 \pm 10 \mathrm{~m}\end{array}$ & 0.15 & 10.46 & $\begin{array}{l}\text { SA(s)bc pec } \\
\text { Sy2.5 } \\
\\
\text { SB0 } 1 \text { pec } \\
\text { LINER }\end{array}$ \\
\hline 22 & $\begin{array}{l}9-22-101 \\
9-22-102\end{array}$ & $\begin{array}{c}\text { ngc } 5278 \\
\text { ugc } 08677 \\
\text { arp } 239 \text { a } \\
\text { vv } 019 \mathrm{a} \\
\text { ngc } 5279 \\
\text { ugc } 08678 \\
\text { arp } 239 \text { b } \\
\text { vv } 019 b\end{array}$ & 133951.80 & 555529.0 & $7580 \pm 46$ o & 0.04 & 14.31 & $\begin{array}{l}\mathrm{SA}(\mathrm{s}) \mathrm{b} ? \text { pec } \\
\mathrm{SB}(\mathrm{s}) \mathrm{a} \text { pec }\end{array}$ \\
\hline 23 & $7-29-48$ & $\begin{array}{l}\text { ngc } 5497 \\
\text { ugc } 09069\end{array}$ & 140826.81 & 390742.7 & $7701 \pm 60$ о & 0.04 & 15.80 & $\overline{\mathrm{SB}(\mathrm{s}) \mathrm{b}}$ \\
\hline 24 & $\begin{array}{l}9-23-64 \mathrm{a} \\
9-23-64 \mathrm{~b}\end{array}$ & $\begin{array}{l}\text { ugc } 09178 \\
\text { arp } 045 \mathrm{a} \\
\text { vv } 002 \mathrm{a} \\
\text { arp } 045 \mathrm{~b} \\
\text { vv 002c }\end{array}$ & $\begin{array}{l}141809.70 \\
141806.96\end{array}$ & $\begin{array}{llll}52 & 07 & 23.4 \\
52 & 07 & 16.3\end{array}$ & $8704 \pm 97$ o & 0.05 & 16.69 & SBbc (l) \\
\hline 25 & $4-35-27$ & $\begin{array}{c}\text { ngc } 5829 \\
\text { ugc } 09673 \text { a } \\
\text { arp } 042 \text { a } \\
\text { vv } 007 \\
\end{array}$ & 150029.05 & 233140.5 & $\begin{array}{c}5716 \pm 38 \text { о } \\
5697 \pm 16 \mathrm{~m}\end{array}$ & 0.19 & 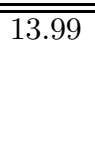 & 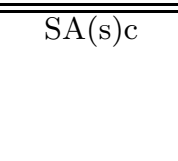 \\
\hline 26 & $10-22-28$ & vv 487 & 153658.10 & $\overline{574554.0}$ & & 0.07 & 16.00 & $\overline{\mathrm{Sc}(\mathrm{l})}$ \\
\hline 27 & $3-41-34$ & $\begin{array}{l}\text { ic } 1162 \\
\text { vv } 452\end{array}$ & 155901.039 & 174900.88 & $\begin{array}{l}13273 \pm 70 \mathrm{o} \\
13326 \pm 9 \mathrm{~m}\end{array}$ & 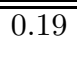 & 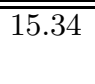 & 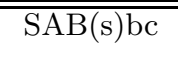 \\
\hline 28 & $\overline{9-27-40}$ & $\begin{array}{c}\text { ugc } 10396 \\
\operatorname{arp} 066 \\
\text { vv } 472\end{array}$ & 162536.66 & $\overline{c 513959.3}$ & $\overline{66185 \pm 96 \text { o }}$ & 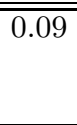 & 15.23 & $\overline{\mathrm{Sbc}}$ \\
\hline 29 & $8-33-14$ & vv 447 & 180304.05 & 473527.9 & & 0.17 & 16.00 & 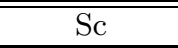 \\
\hline 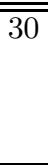 & & $\begin{array}{c}\text { ugc } 11680 \mathrm{a} \\
\text { vv } 476 \mathrm{a} \\
\text { ugc } 11680 \mathrm{~b} \\
\text { vv } 476 \mathrm{~b} \\
\end{array}$ & $\begin{array}{lll}21 & 05 & 10.70 \\
21 & 05 & 15.071\end{array}$ & $\begin{array}{l}034015.0 \\
034031.86\end{array}$ & $\begin{array}{c}7813 \pm 117 \mathrm{o} \\
7767 \pm 9 \mathrm{~m} \\
7897 \pm 1\end{array}$ & $\begin{array}{l}0.36 \\
0.36\end{array}$ & $\begin{array}{l}14.20 \\
14.50\end{array}$ & $\begin{array}{c}\text { Sa } \\
\\
\text { Compact } \\
\text { Sy2 } \\
\end{array}$ \\
\hline 31 & $\begin{array}{l}5-56-5 \\
5-56-4\end{array}$ & $\begin{array}{c}\text { ngc } 7753 \\
\text { ugc } 12780 \\
\text { arp } 086 \mathrm{a} \\
\text { vv } 005 \mathrm{a} \\
\text { ngc } 7752 \\
\text { ugc } 12779 \\
\text { arp } 086 \mathrm{~b} \\
\text { vv } 005 \mathrm{~b}\end{array}$ & 234427.10 & 291052.0 & $\begin{array}{l}5185 \pm 83 \mathrm{o} \\
5164 \pm 5 \mathrm{~m}\end{array}$ & 0.42 & 13.86 & $\overline{\mathrm{SAB}(\mathrm{rs}) \mathrm{bc}}$ \\
\hline 32 & $\overline{~ 1-60-37}$ & $\begin{array}{c}\text { ngc } 7757 \\
\text { ugc } 12788 \\
\text { arp } 068 \\
\text { vv } 407 \\
\end{array}$ & 20234611.60 & 035343.0 & $2955 \pm 5 \mathrm{r}$ & 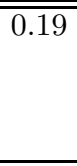 & 13.26 & $\overline{\mathrm{SA}(\mathrm{rs}) \mathrm{c}}$ \\
\hline
\end{tabular}


Table 8. Catalog of M 51-type galaxies - the initial data.

\begin{tabular}{|c|c|c|c|c|c|c|c|c|}
\hline \multicolumn{9}{|c|}{ GALAXIES OF TYPE M 51 (THE SAMPLE TW) } \\
\hline$N$ & $\overline{M C G}$ & Names & $\mathrm{RA}(1950.0)$ & $\overline{\text { DEC }(1950.0)}$ & $V_{\mathrm{r}}$ & $A_{B}$ & $B_{\mathrm{T}}$ & Type \\
\hline 1 & $-2-12-38$ & vV 468 & 043039.70 & -105502.1 & & 0.16 & 15.22 & Sc (l) \\
\hline 2 & $5-21-13$ & vv 473 & 084758.7 & 292210.0 & $7994 \pm 82$ о & 0.16 & 16.10 & $\overline{\mathrm{SBb}(\mathrm{l})}$ \\
\hline 3 & $1-31-9$ & vv 462 & 115910.60 & 060559.0 & $\begin{array}{c}1346 \pm 57 \mathrm{o} \\
1350 \pm 10 \mathrm{~m}\end{array}$ & 0.06 & $\begin{array}{c}14.75 \\
16.5\end{array}$ & \\
\hline 4 & $\begin{array}{l}5-31-154 \mathrm{a} \\
5-31-154 \mathrm{~b}\end{array}$ & vv 474 & $\begin{array}{lll}13 & 10 & 36.36 \\
13 & 10 & 35.69 \\
\end{array}$ & $\begin{array}{llll}27 & 24 & 21.3 \\
27 & 24 & 01.1 \\
\end{array}$ & $6863 \pm 60$ o & $\begin{array}{l}0.05 \\
0.05\end{array}$ & $\begin{array}{c}15.65 \\
16.5\end{array}$ & $\begin{array}{l}\mathrm{SBc} \\
\mathrm{E} ?\end{array}$ \\
\hline 5 & $3-38-7$ b & $\begin{array}{l}\text { ugc } 09503 \mathrm{a} \\
\operatorname{arp} 064 \mathrm{a} \\
\text { vv } 471 \mathrm{a} \\
\text { ugc } 09503 \mathrm{~b} \\
\operatorname{arp} 064 \mathrm{~b} \\
\text { vv } 471 \mathrm{~b} \\
\text { ugc } 09503 \mathrm{c} \\
\operatorname{arp} 064 \mathrm{c} \\
\text { vv } 471 \mathrm{c}\end{array}$ & 144309.00 & 194106.0 & $\begin{array}{l}9396 \pm 60 \mathrm{o} \\
9401 \pm 8 \mathrm{~m}\end{array}$ & 0.17 & 15.00 & $\mathrm{Sb}$ \\
\hline 6 & $-2-59-14$ & vv 461 & 232103.12 & -083408.4 & $15877 \pm 60$ о & 0.17 & 15.79 & Sb (l) \\
\hline
\end{tabular}

Table 9. Catalog of M 51-type galaxies - the derived data.

\begin{tabular}{|c|c|c|c|c|c|c|c|c|c|c|c|c|}
\hline \multicolumn{13}{|c|}{ GALAXIES OF TYPE M 51 (THE SAMPLE G) } \\
\hline$N$ & $B_{\mathrm{sat}}^{\mathrm{T}}$ & $D$ & $M_{\text {main }}^{B}$ & $L_{B}\left(L_{\odot}\right)$ & $M_{\text {sat }}^{B}$ & $\frac{L_{\text {sat }}}{L_{\text {main }}}$ & $\frac{\mathrm{ma}}{\mathrm{sep}}$ & $Q_{1}$ & $L_{\mathrm{FIR}}\left(L_{\odot}\right)$ & $S F R$ & $\frac{L_{\mathrm{FIR}}}{L_{B}}$ & $d \times d$ \\
\hline 1 & 17.38 & 79.7 & -19.76 & $6.17 \times 10^{9}$ & -17.26 & 0.10 & 1.36 & -2.61 & $4.72 \times 10^{9}$ & 2.5 & 0.6 & $1.34 \times 1.34$ \\
\hline 2 & 15.99 & 49.4 & -21.36 & $2.70 \times 10^{10}$ & -17.62 & 0.03 & 1.29 & -3.33 & $9.16 \times 10^{9}$ & 4.8 & 0.3 & $3.91 \times 3.91$ \\
\hline 3 & 15.65 & 104.7 & -20.90 & $1.77 \times 10^{10}$ & -19.57 & 0.29 & 0.65 & -1.98 & $6.02 \times 10^{10}$ & 31.3 & 2.8 & $2.78 \times 2.78$ \\
\hline 4 & 16.90 & 77.1 & -20.80 & $1.62 \times 10^{10}$ & -17.77 & 0.06 & 0.76 & -1.70 & $3.39 \times 10^{9}$ & 1.8 & 0.2 & $2.21 \times 2.21$ \\
\hline 5 & 16.51 & 172.5 & -21.28 & $2.52 \times 10^{10}$ & -19.79 & 0.25 & 0.52 & -2.20 & $2.66 \times 10^{10}$ & 13.8 & 0.9 & $1.34 \times 1.34$ \\
\hline 6 & 16.30 & 21.4 & -17.91 & $1.12 \times 10^{9}$ & -15.51 & 0.11 & 0.78 & -2.69 & $3.17 \times 10^{8}$ & 0.2 & 0.2 & $2.32 \times 2.32$ \\
\hline 7 & 14.70 & 55.3 & -20.45 & $1.17 \times 10^{10}$ & -19.19 & 0.31 & 0.62 & -4.13 & $1.43 \times 10^{10}$ & 7.5 & 1.0 & $3.40 \times 3.40$ \\
\hline 8 & 18.05 & & & & & 0.07 & 1.23 & -5.28 & & & & $0.91 \times 0.91$ \\
\hline 9 & 17.43 & 46.8 & -18.29 & $1.60 \times 10^{9}$ & -16.13 & 0.14 & 0.83 & -5.23 & $2.81 \times 10^{9}$ & 1.5 & 1.4 & $1.33 \times 1.33$ \\
\hline 10 & 17.43 & 162.6 & -20.90 & $1.76 \times 10^{10}$ & -18.75 & 0.14 & 0.68 & -3.75 & $3.93 \times 10^{10}$ & 20.5 & 1.8 & $2.78 \times 2.78$ \\
\hline 11 & 15.88 & 125.7 & -21.36 & $2.70 \times 10^{10}$ & -19.82 & 0.24 & 0.52 & -4.31 & $2.77 \times 10^{10}$ & 14.4 & 0.8 & $2.98 \times 2.98$ \\
\hline 12 & 19.11 & 87.8 & -18.34 & $1.67 \times 10^{9}$ & -15.66 & 0.09 & 0.61 & -2.36 & & & & $1.33 \times 1.33$ \\
\hline 13 & 14.40 & 44.7 & -19.63 & $5.49 \times 10^{9}$ & -18.98 & 0.55 & 0.57 & -1.85 & & & & $2.98 \times 2.98$ \\
\hline 14 & 14.40 & 95.4 & -20.82 & $1.64 \times 10^{10}$ & -20.61 & 0.82 & 0.43 & -2.74 & $4.79 \times 10^{10}$ & 24.9 & 2.4 & $2.55 \times 2.55$ \\
\hline 15 & 16.98 & 80.8 & -20.21 & $9.35 \times 10^{9}$ & -17.65 & 0.09 & 0.89 & -2.20 & $3.12 \times 10^{10}$ & 16.2 & 2.7 & $1.90 \times 1.90$ \\
\hline 16 & 14.42 & 12.8 & -19.46 & $4.71 \times 10^{9}$ & -16.20 & 0.05 & 0.99 & -1.34 & $6.80 \times 10^{9}$ & 3.5 & 1.2 & $4.45 \times 4.45$ \\
\hline 17 & 18.06 & 140.6 & -20.44 & $1.16 \times 10^{10}$ & -17.74 & 0.08 & 0.58 & -1.49 & & & & $1.62 \times 1.62$ \\
\hline 18 & 18.40 & 55.8 & -19.40 & $4.46 \times 10^{9}$ & -15.54 & 0.03 & 1.75 & -1.58 & $8.66 \times 10^{9}$ & 4.5 & 1.6 & $1.19 \times 1.19$ \\
\hline 19 & 17.55 & 10.0 & -16.04 & $2.01 \times 10^{8}$ & -12.56 & 0.04 & 0.76 & -2.07 & & & & $2.01 \times 2.01$ \\
\hline 20 & 18.98 & & & & & 0.06 & 0.67 & -1.32 & & & & $1.19 \times 1.19$ \\
\hline 21 & 10.46 & 9.0 & -20.97 & $1.89 \times 10^{10}$ & -19.45 & 0.25 & 0.97 & -0.80 & $7.03 \times 10^{9}$ & 3.7 & 0.3 & $14.20 \times 14.20$ \\
\hline 22 & 14.91 & 104.0 & -20.81 & $1.64 \times 10^{10}$ & -20.21 & 0.58 & 0.62 & -1.46 & $3.58 \times 10^{10}$ & 18.6 & 1.8 & $2.32 \times 2.32$ \\
\hline 23 & 18.25 & 105.3 & -19.35 & $4.26 \times 10^{9}$ & -16.90 & 0.10 & 1.02 & -1.31 & & & & $0.91 \times 0.91$ \\
\hline 24 & 18.97 & 119.2 & -18.74 & $2.42 \times 10^{9}$ & -16.47 & 0.12 & 0.67 & -0.88 & $6.79 \times 10^{9}$ & 3.5 & 2.3 & $1.33 \times 1.33$ \\
\hline 25 & 17.38 & 77.9 & -20.66 & $1.42 \times 10^{10}$ & -17.27 & 0.04 & 0.43 & -2.56 & $1.24 \times 10^{10}$ & 6.4 & 0.7 & $1.70 \times 1.70$ \\
\hline 26 & 18.35 & & & & & 0.11 & 1.08 & -3.95 & & & 1.1 & $0.88 \times 0.88$ \\
\hline 27 & 18.64 & 179.6 & -21.12 & $2.17 \times 10^{10}$ & -17.82 & 0.05 & 0.73 & -3.44 & $3.28 \times 10^{10}$ & 17.0 & 1.2 & $1.33 \times 1.33$ \\
\hline 28 & 17.97 & 85.9 & -19.53 & $5.01 \times 10^{9}$ & -16.79 & 0.08 & 1.45 & -3.92 & $8.74 \times 10^{9}$ & 4.5 & 1.4 & $1.33 \times 1.33$ \\
\hline 29 & 18.48 & & & & & 0.10 & 1.63 & -3.69 & & & & $1.19 \times 1.19$ \\
\hline 30 & 14.50 & 104.7 & -21.26 & $2.47 \times 10^{10}$ & -20.96 & 0.76 & 0.81 & -3.29 & $5.14 \times 10^{10}$ & 26.7 & 1.7 & $2.83 \times 2.83$ \\
\hline 31 & 14.97 & 70.9 & -20.81 & $1.64 \times 10^{10}$ & -19.70 & 0.36 & 0.46 & -3.73 & $4.45 \times 10^{10}$ & 23.1 & 2.2 & $0.79 \times 0.79$ \\
\hline 32 & 17.57 & 40.0 & -19.94 & $7.30 \times 10^{9}$ & -15.63 & 0.02 & 1.04 & -2.82 & $3.85 \times 10^{9}$ & 2.0 & 0.4 & $2.32 \times 2.32$ \\
\hline
\end{tabular}


0.4 only (de Jong et al. 1984). Therefore, we suppose the high rate of star formation in M 51-type galaxies.

In Fig. 5 we plot the FIR luminosities of the main galaxies versus their blue luminosities. The solid line in this figure represents the smoothed relation for interacting spirals according to Bushouse et al. (1988). As one can see, the M 51-type objects follow the same $L_{\mathrm{FIR}}-L_{B}$ correlation as for interacting galaxies. To more clearly define the influence of spatial environment on FIR luminosity, we introduced the value of $Q_{1}=\log Q=-2.6$ that divides the $\mathrm{G}$ sample into two equal-size subsamples (sixteen galaxies each). One can note that relatively isolated systems $\left(Q_{1}<-2.6\right)$ and systems in a more dense spatial environment $\left(Q_{1}>-2.6\right)$ demonstrate the same realation.

Many authors tried to find any links between $S F R$ and different characteristics of galaxies (see, for instance, Kennicutt 1998; Tomita \& Tomita 1996). Numerous researches have showed that $S F R$ enhancement is connected to the mutual influence of components in systems. Since the tidal force is propotional to the mass of the satellite and the mass of the satellite is propotional to the luminosity, we can consider the luminosity of satellite to luminosity of main galaxy ratio (denoted by $\mathrm{F}$ above) as characteristic of the interaction force between galaxies. Figure 6 presents the dependence of $S F R$ on F.

A trend of values of $S F R$ with $\mathrm{F}$ can be seen. Subsamples of M 51-type galaxies in different spatial environments show the same trends so the local mutual interaction is more essential than the global one. Thus, we can suppose the systems with the relatively brighter (more massive) satellites demonstrate the most considerable star formation. The values of the coefficients of linear correlation of these dependencies are $R_{S F R}=0.68$ (23 galaxies), $R_{L_{\mathrm{FIR}} / L_{B}}=0.42$ (24 galaxies). After exclusion of three galaxies with the largest $L_{\mathrm{s}} / L_{\mathrm{m}}$ ratios (VV 476 , NGC 5278, and Arp 87), the correlation coefficients are decreased to 0.55 (the significance of the correlation is $99 \%$ ) and 0.29 for the $S F R$ and $L_{\mathrm{FIR}} / L_{B}$ correspondingly. Therefore, the interperetation of these dependencies have to be carried out with a strong caution.

Some authors discussed the link of FIR luminosity with the morphology of galaxies. For example, Tomita \& Tomita (1996) found a weak trend of $L_{\mathrm{FIR}} / L_{B}$ ratio with the bar presence. We compared the SFR and $L_{\mathrm{FIR}} / L_{B}$ values for barred and non-barred galaxies and found that the mean characteristics for two subsamples are consistent within errors.

At last we found a significant correlation between $S F R$ and redshift. The value of the correlation coefficient is 0.55 (23 galaxies). Kennicutt (1998) pointed out this dependence as well. It might be explained by selection effects because the fraction of galaxies with high luminosity (and usually with enchanced star formation) increases with $z$.

\section{Conclusions}

The main results of our study are

1. We have compiled a new sample of 32 binary systems consisting of the most typical M 51-type galaxies. From the statistical analysis of the sample we have proposed a formal definition of such binaries: M 51-type galaxies are systems with a faint companion (with blue luminosity between $1 / 30$ and $1 / 3$ of the main galaxy) near the end (at less than 2 main galaxy diameters projected distance) of a spiral arm of the primary galaxy.

2. As a rule, the satellites are situated near the boundary of the stellar disks of the main galaxies that can testify to the existence of the circular stationary orbits according to some theoretical arguments.

3. The main galaxies demonstate an excess of FIR luminosity. Probably, this means that star formation in M 51-type galaxies increased. The trend of the values of $S F R$ and $L_{\mathrm{FIR}} / L_{B}$ on the luminosities of components ratio is suspected - systems with relatively brighter (more massive) satellites show the strongest star formation. Because of the relatively small sample size, this conclusion is tentative only.

Our sample gives a direct basis for future work on this specific type of binary system.

Acknowledgements. We would like to thank the referees for useful remarks and suggestions. This work was supported in part by the "Integration" programme (A0145). We have used the Lyon-Meudon Extragalactic Database (LEDA) supplied by the LEDA team at the CRAL-Observatoire de Lyon (France) and the NASA/IPAC Extragalactic Database (NED) which is operated by the Jet Propulsion Laboratory, California Institute of Technology, under contract with the National Aeronautics and Space Administration.

\section{Appendix A}

Catalogs of the initial data. The columns are as follows: Column (1) - Ordinal number of galaxy;

Column (2) - Galaxy identification by MCG;

Column (3) - Galaxy identification by other catalogs;

Column (4) - Right ascension, according to the NED;

Column (5) - Declination, according to the NED;

Column (6) - Heliocentric radial velocities, in $\mathrm{km} \mathrm{s}^{-1}$, according to the LEDA, where o - designation of the velocity defined from optical observations, $r$ - velocity from radio observations, $m$ - the mean value of velocity defined from optical and radio observations;

Column (7) - Blue Galactic extinction, according to the NED, in magnitudes;

Column (8) - Total apparent $B$ magnitude, taken from the LEDA;

Column (9) - Morphological type, according to the NED, in brackets-according to the LEDA.

Catalogs of the derived data. The columns are as follows: Column (1) - Ordinal number of the system according to the tables of initial data (see Tables 7 and 8);

Column (2) - Total apparent $B$ magnitude of the satellite; Column (3) - Adopted distance, in Mpc;

Column (4) - Total absolute $B$ magnitude of the main galaxy;

Column (5) - Luminosity of the main galaxy, in solar units $\left(M_{\odot}=4.72\right)$; 


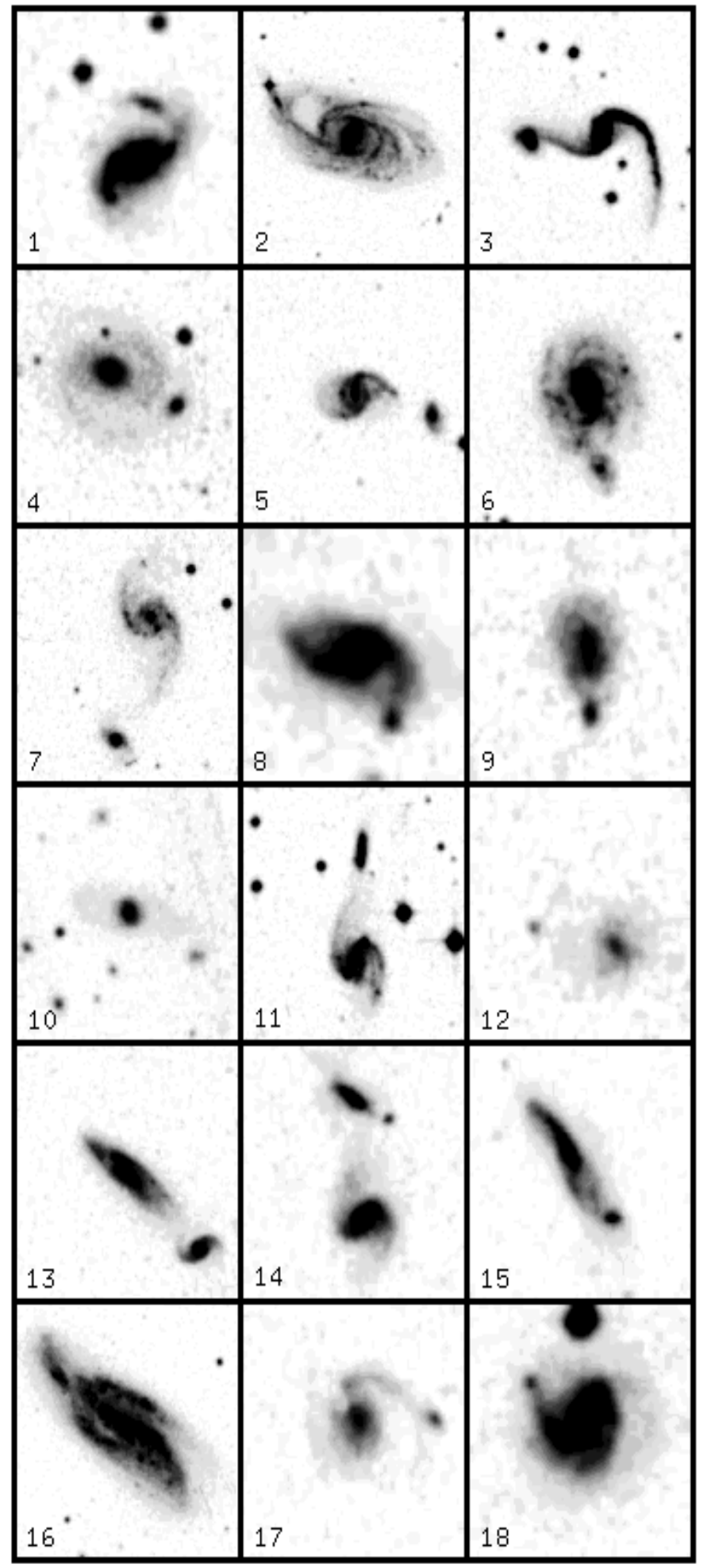

Fig. 7. Digital Sky Survey images of M 51 type galaxies. The size of each image in arcmin is indicated in the last column of Table 9. North is top, East is to the left. The images are marked in accordance with the first column of Tables 7-10.

Column (6) - Total absolute $B$ magnitude of the satellite; Column (7) - The luminosity of satellite to luminosity of main galaxy ratio;

Column (8) - The semimajor axis to angular separation ratio;

Column (9) - The interaction strength $Q_{1}=\log Q$;
Column (10) - FIR luminosity of the system, in solar units;

Column (11) - Star formation rate, in $M_{\odot} / \mathrm{yr}$;

Column (12) - The $L_{\mathrm{FIR}}$ to $L_{B}$ ratio;

Column (13) - Size of image area in the atlas of type M 51 galaxies, in arcmin. 
Table 10. Catalog of M 51-type galaxies - the derived data.

\begin{tabular}{|c|c|c|c|c|c|c|c|c|c|c|c|c|}
\hline \multicolumn{13}{|c|}{ GALAXIES OF TYPE M 51 (THE SAMPLE TW) } \\
\hline$N$ & $B_{\text {sat }}^{\mathrm{T}}$ & $D$ & $M_{\text {main }}^{B}$ & $L_{B}\left(L_{\odot}\right)$ & $M_{\mathrm{sat}}^{B}$ & $\frac{L_{\text {sat }}}{L_{\text {main }}}$ & $\frac{\mathrm{ma}}{\mathrm{sep}}$ & $Q_{1}$ & $L_{\mathrm{FIR}}\left(L_{\odot}\right)$ & $S F R$ & $\frac{L_{\mathrm{FIR}}}{L_{B}}$ & $d \times d$ \\
\hline 1 & $\begin{array}{l}19.31 \\
18.35\end{array}$ & & & & & $\begin{array}{l}0.02 \\
0.06\end{array}$ & $\begin{array}{l}0.76 \\
0.89\end{array}$ & -3.85 & & & & $1.90 \times 1.90$ \\
\hline 2 & $\begin{array}{l}16.83 \\
17.88 \\
\end{array}$ & 107.6 & -19.22 & $3.76 \times 10^{9}$ & $\begin{array}{r}-18.49 \\
-17.44 \\
\end{array}$ & $\begin{array}{l}0.51 \\
0.19\end{array}$ & $\begin{array}{l}0.97 \\
0.58 \\
\end{array}$ & -1.12 & & & & $1.19 \times 1.19$ \\
\hline 3 & $\begin{array}{l}18.02 \\
18.70\end{array}$ & 18.1 & -16.60 & $3.83 \times 10^{8}$ & $\begin{array}{l}-13.33 \\
-12.66\end{array}$ & $\begin{array}{l}0.05 \\
0.03\end{array}$ & $\begin{array}{l}1.29 \\
0.72\end{array}$ & -3.45 & & & & $0.87 \times 0.87$ \\
\hline 4 & $\begin{array}{l}16.50 \\
17.12 \\
\end{array}$ & 93.3 & -19.25 & $3.87 \times 10^{9}$ & $\begin{array}{r}-18.40 \\
-17.77 \\
\end{array}$ & $\begin{array}{l}0.46 \\
0.26\end{array}$ & $\begin{array}{l}0.60 \\
0.89 \\
\end{array}$ & -2.92 & $1.32 \times 10^{10}$ & 6.9 & 2.8 & $1.33 \times 1.33$ \\
\hline 5 & $\begin{array}{l}17.79 \\
18.67\end{array}$ & 127.1 & -20.69 & $1.46 \times 10^{10}$ & $\begin{array}{l}-17.90 \\
-17.02\end{array}$ & $\begin{array}{l}0.08 \\
0.03\end{array}$ & $\begin{array}{l}1.16 \\
0.85\end{array}$ & -1.94 & $1.14 \times 10^{10}$ & 5.9 & 0.6 & $1.98 \times 1.98$ \\
\hline 6 & $\begin{array}{l}17.02 \\
18.79\end{array}$ & 211.6 & -21.01 & $1.95 \times 10^{10}$ & $\begin{array}{l}-19.78 \\
-18.01\end{array}$ & $\begin{array}{l}0.32 \\
0.06\end{array}$ & $\begin{array}{l}0.78 \\
0.86\end{array}$ & -5.26 & & & & $1.33 \times 1.33$ \\
\hline
\end{tabular}

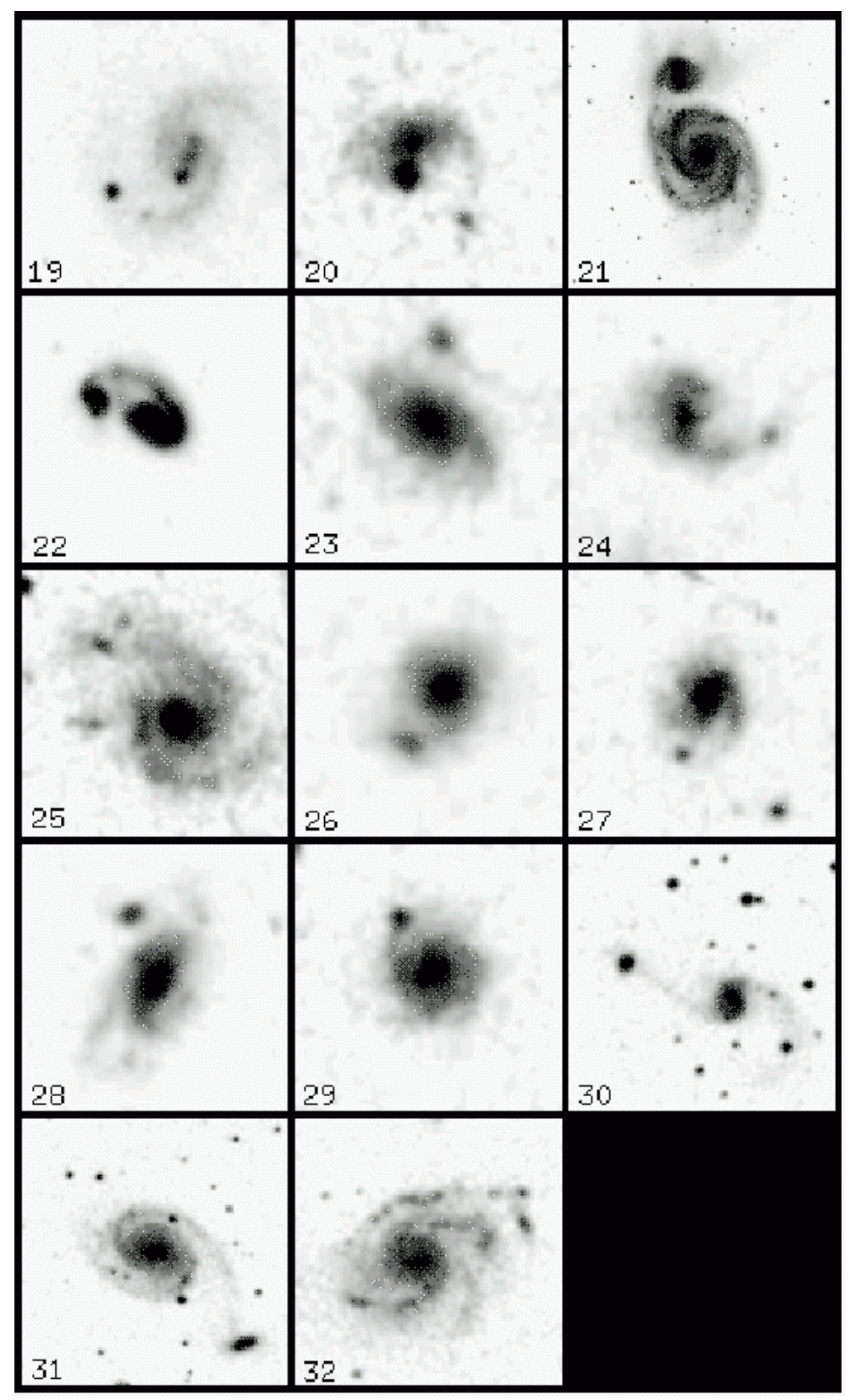

Fig. 8. Continuation of Fig. 7. 


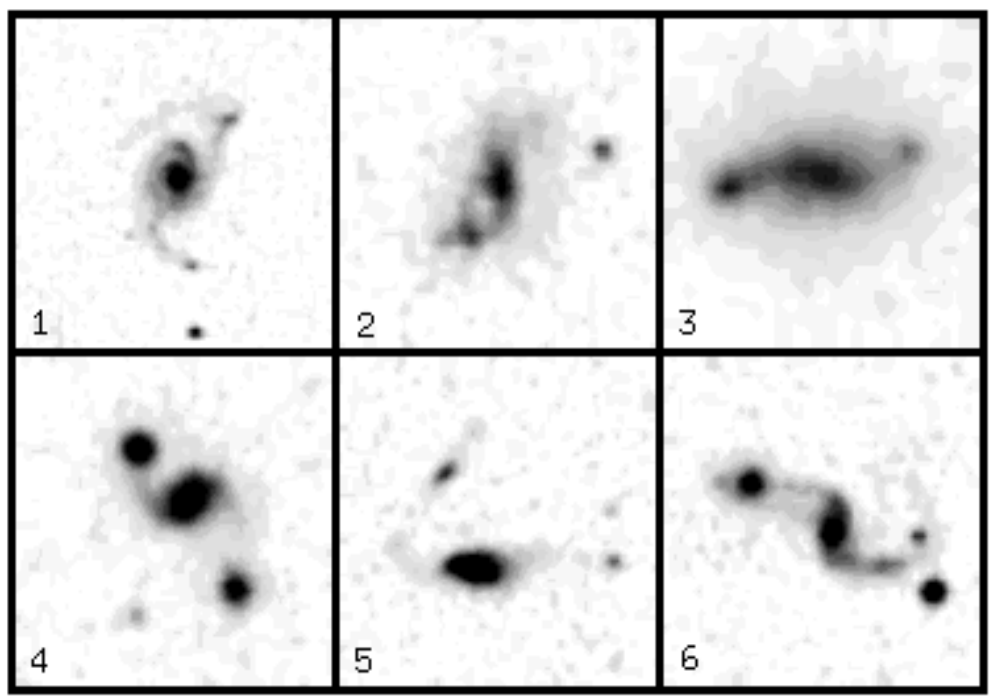

Fig. 9. The same as Fig. 7 but for twice M 51 type galaxies.

\section{Appendix B}

Here we present the atlas of M 51 type galaxies for the both samples.

\section{References}

Arp, H. 1966, ApJS, 14, 123

Bushouse, H. 1987, AJ, 320, 49

Bushouse, H., Lamb, S. A., \& Werner, M. W. 1988, AJ, 335, 74

Dahari, O. 1984, AJ, 89, 966

de Jong, T., Clegg, P. E., Soifer, B. T., et al. 1984, ApJ, 278, $67 \mathrm{~L}$

de Vaucouleurs, G. 1963, ApJS, 8, 31

de Vaucouleurs, G., de Vaucouleurs, A., Corwin, H. G., et al. 1991, The Third Reference Catalogue of Bright Galaxies (Springer-Verlag, New York)

Elmegreen, D. M., Elmegreen, B. G., \& Bellin, A. D. 1990, ApJ, 364, 415

Eskridge, P. B., Frogel, J. A., Pogge, R. W., et al. 2000, AJ, 119,536

Felten, J. E. 1976, ApJ, 207, 700

Hu, G., \& Sulentic, J. 1991, ApJ, 374, 407
Huchra, J., \& Sargent, W. 1973, AJ, 186, 433

Howard, S., \& Byrd, G. 1990, AJ, 99, 1978

Karachentsev, I. D. 1972, Soobsch. SAO, 7, 3

Karachentsev, I. D. 1987, Binary galaxies (Moscow, Nauka)

Keel, W. 1993, AJ, 106, 1771

Kennicutt, R. 1998, ARA\&A, 36, 189

Laurikainen, E., Salo, H., \& Aparicio, A. 1998, A\&AS, 129, 517

Lin, D., \& Tremaine, S. 1983, ApJ, 264, 364

Marzke, R. O., da Costa, L. N., Pellegrini, P. S., et al. 1998, ApJ, 503, 617

Pasha, I. I., Polyachenko, V. L., \& Strelnikov, A. V. 1988, Pis'ma v AZh, 14, 195

Salo, H., \& Laurikainen, E. 1993, AJ, 410, 586

Schmidt, M. 1968, ApJ, 151, 393

Sharp, N. A. 1986, PASP, 98, 740

Tomita, A., Tomita, Y., \& Saito, M. 1996, PASJ, 48, 285

Toomre, A., \& Toomre, J. 1972, ApJ, 178, 623

Vorontsov-Velyaminov, B. A. 1957, AZh, 34, 8

Vorontsov-Velyaminov, B. A. 1975, AZh, 52, 692

Vorontsov-Velyaminov, B. A. 1977, A\&AS, 28, 1

Vorontsov-Velyaminov, B. A., et al. 1962-1968, Morphological Catalog of Galaxies, vols. 1-4, MSU

Xu, C., \& Sulentic, J. W. 1991, ApJ, 374, 407 